\title{
Opuntia humifusa modulates morphological changes characteristic of asthma via IL-4 and IL-13 in an asthma murine model
}

\author{
Soon-Young Lee ${ }^{a}$, Chun-Sik Bae ${ }^{b}$, Young-hoon Choic, Nam-Sook Seo ${ }^{a}$, Chang-Su Na ${ }^{a}$, Jin-Cheol Yoo ${ }^{d}$ \\ Seung Sik Cho ${ }^{c}{ }^{c}$ and Dae-Hun Park ${ }^{a}$
}

${ }^{a}$ College of Oriental Medicine, Dongshin University, Naju, Jeonnam, Korea; ${ }^{b}$ College of Veterinary Medicine, Chonnam National University, Gwangju Korea; 'Department of Pharmacy, College of Pharmacy, Mokpo National University, Muan, Jeonnam, Korea; ${ }^{\mathrm{d} D e p a r t m e n t}$ of Pharmacy, College of Pharmacy, Chosun University, Gwangju, Korea

\begin{abstract}
Asthma is a chronic pulmonary disease that affects an estimated 235 million people worldwide, but asthma drugs have many adverse effects. Opuntia humifusa (eastern prickly pear) has been used as a food and traditional medicine worldwide; however, its anti-asthmatic effects have not been reported. We evaluated $O$. humifusa as a potential therapeutic or preventive component of antiasthmatic drugs. We divided ovalbumin-sensitized mice into the following groups: normal control, asthma-induced control, dexamethasone-treated group (positive control), $50 \mathrm{mg} / \mathrm{kg} \mathrm{O}$. humifusatreated group, $100 \mathrm{mg} / \mathrm{kg} \mathrm{O}$. humifusa-treated group, and $500 \mathrm{mg} / \mathrm{kg} \mathrm{O}$. humifusa-treated group. Levels of Th1/Th2/Th17-related cytokines were evaluated using RT-PCR, ELISA, and immunohistochemistry. O. humifusa dose-dependently suppressed the morphological changes typically observed in asthma, such as goblet cell hyperplasia, inflammatory cell infiltration, mucous hypersecretion, and relative basement membrane thickening in the respiratory system. These results may be attributable to regulation of Th1-/Th2-/Th17-related factors, especially interleukin (IL)-4 and IL13. We conclude that $O$. humifusa is a potential anti-asthmatic functional food.

Abbreviations: O. humifusa: Opuntia humifusa; Th: helper T; RT-PCR: real-time polymerase chain reaction; ELISA: enzyme-linked immunosorbent assay; IL: interleukin; WHO: World Health Organization; IFN- $\gamma$ : interferon gamma; TNF-a: tumor necrosis factor-alpha; IgE: immunoglobulin E; CD: cluster of differentiation; OVA: ovalbumin; DEX: dexamethasone; BALF: bronchoalveolar fluid; H\&E: hematoxylin and eosin; PAS: periodic acid-schiff; PBS: phosphate-buffered saline; BM: basement membrane; CDNA: complementary deoxyribonucleic acid; RNA: ribo nucleic acid; RIPA: radioimmunoprecipitation assay; IHC: immunohistochemistry; HPLC: high-performance liquid chromatography; SD: standard deviation; WBC: white blood cells; APCs: antigen-presenting cells
\end{abstract}

\section{ARTICLE HISTORY}

Received 16 August 2017

Accepted 11 October 2017

\section{KEYWORDS}

Opuntia humifusa;

ovalbumin-induced asthma; IL-4; IL-13

\section{Introduction}

Asthma is a chronic disease related to the pulmonary system; the number of asthma patients worldwide was estimated at 235 million in 2013. Asthma's many inducers are referred to as allergens, and include pollen, pet dander, dust mites, tobacco smoke, and environmental pollutants [1]. Asthma is an incurable chronic disease; its typical symptoms vary from cough to obstructive apnea caused by mucous hypersecretion, epithelial hyperplasia, basement membrane thickening, and inflammatory cell infiltration near bronchioles and vessels $[2,3]$.

It has been shown that asthma is the result of a Th1/ Th2 imbalance [2]. Th1-related cytokines include interferon gamma (IFN- $\gamma$ ), which affects the severity of asthma [4], and interleukin 12 (IL-12), which induces
Th1 cells and suppresses Th2 cells [5,6]. Th2-related cytokines include IL-4, IL-5, IL-13, and Th17-related cytokines (tumor necrosis factor-alpha (TNF- $\alpha$ ) IL-6, and IL-1 $\beta$ ) [7]. IL-4 modulates IgE levels, resulting in inflammatory cell migration to inter-respiratory cells [8], and IL-13 induces morphological changes in the pulmonary system typical of asthma, such as mucous hypersecretion, epithelial hyperplasia, base membrane thickening, inflammatory cell infiltration, and B cell activation [9-12]. TNF- $\alpha$ is produced by macrophages and is involved in the interaction between mast cells [13] and airway smooth muscle cells, a key mechanism for inducing airway hyperresponsiveness [14]. In asthma, IL-6 is upregulated in pulmonary epithelial cells by various stimuli [15] and is involved in facilitating IL-4 differentiation, downregulating Th2 cell differentiation, and

CONTACT Seung Sik Cho sscho@mokpo.ac.kr Department of Pharmacy, College of Pharmacy, Mokpo National University, Muan, Jeonnam 58554, Korea; Dae-Hun Park dhj1221@paran.com E College of Oriental Medicine, Dongshin University, Naju, Jeonnam 58245, Korea

(1) Supplemental data for this article can be accessed here.

(c) 2017 The Author(s). Published by Informa UK Limited, trading as Taylor \& Francis Group.

This is an Open Access article distributed under the terms of the Creative Commons Attribution License (http://creativecommons.org/licenses/by/4.0/), which permits unrestricted use, distribution, and reproduction in any medium, provided the original work is properly cited. 
promoting Th17 cell differentiation [16]. Mast cells respond to inflammatory inducers in the early phase and release TNF- $\alpha$ to bring on typical asthma characteristics, such as contracting smooth muscles [14], attracting neutrophils and eosinophils [17], and activating T cells [18]. IL-6 is an important factor that regulates asthma induction via CD4+ cell modulation [19].

Although inhaled corticosteroids have been commonly used for suppressing typical asthma symptoms [20], they can cause various adverse health effects such as growth defects in children [21], cataracts and glaucoma, hypertension, hyperlipidemia, peptic ulcers, myopathy, and immunological suppression [22]. These issues underscore the need to develop new anti-asthmatic drugs with few or no adverse effects.

Opuntia humifusa (O. humifusa, eastern prickly pear) has been used as a food worldwide. Recently, it has been shown to have antifungal [23] and anticancer $[24,25]$ properties, as well as to improve insulin sensitivity [26] and increase bone density [27]. The objective of this study was to analyze the possible anti-asthmatic effects of O. humifusa and their mechanisms.

\section{Materials and methods}

\section{O. humifusa preparation}

O. humifusa leaves were provided by Jeollanamdo Wando Arboretum, in Jeonnam, Korea. A voucher specimen (MNUCSS-OH-01) was deposited at Mokpo National University (Muan, Korea). The leaves were separated for the present study. Air-dried and powdered $O$. humifusa leaves $(10 \mathrm{~g})$ were extracted twice with $80 \%$ ethanol $(100 \mathrm{~mL})$ at room temperature for 3 days. The resultant $80 \%$ ethanol solution was evaporated, dried, and stored at $-50^{\circ} \mathrm{C}$.

\section{Nutritional content}

A $100 \mathrm{~g}$ sample of $O$. humifusa extract was analyzed for nutritional components by the Jeonnam Biofood Technology Center (Jeonnam, Korea). The extract was analyzed for carbohydrate, fat, cholesterol, and protein. All tests performed were in compliance with the standards recommended by the Association of Analytical Communities/Association of Official Agricultural Chemist and American Association of Cereal Chemists.

\section{Animal experiments}

Using the same methods, two animal studies were conducted at different times. Eighty-four female BALB/c mice were purchased from Samtako (Osan, Korea) and divided into six groups according to treatment: (1) vehicle control (sterilized tap water), (2) ovalbumin (OVA)induced asthma model, (3) $1 \mathrm{mg} / \mathrm{kg} /$ day dexamethasone with OVA induction, (4) $50 \mathrm{mg} / \mathrm{kg} /$ day O. humifusa with OVA induction, (5) $100 \mathrm{mg} / \mathrm{kg} /$ day O. humifusa with OVA induction, and (6) $500 \mathrm{mg} / \mathrm{kg} /$ day O. humifusa with OVA induction. On days 1 and 8 , all mice except those used as the vehicle control were sensitized via intraperitoneal injections of $20 \mu \mathrm{g}$ OVA (Sigma-Aldrich, St. Louis, MO, USA) and $1 \mathrm{mg}$ aluminum hydroxide hydrate (Sigma-Aldrich) in $500 \mu \mathrm{L}$ saline. From day 21 to day 25 , the mice were challenged once daily with $5 \%$ OVA for $30 \mathrm{~min}$ using a nebulizer $(3 \mathrm{~mL} / \mathrm{min}$, NE-U17, Omron, Kyoto, Japan). During the same 5-day period, the treatment groups were also treated once daily with oral doses of sterilized tap water, dexamethasone, $50 \mathrm{mg} / \mathrm{kg} /$ day O. humifusa, $100 \mathrm{mg} / \mathrm{kg} /$ day O. humifusa, or $500 \mathrm{mg} /$ $\mathrm{kg}$ /day O. humifusa $1 \mathrm{~h}$ prior to the OVA challenge. The mice in the vehicle control group were sensitized with OVA according to the same procedure as the other groups of mice ( $20 \mu \mathrm{g}$ OVA and $1 \mathrm{mg}$ aluminum hydroxide hydrate in $500 \mu \mathrm{L}$ saline), after which they were exposed to saline and aluminum hydroxide hydrate by a nebulizer for 5 consecutive days.

\section{Ethics statement}

All experiments were approved by the Institutional Animal Care and Use Committee at Chonnam National University (Animal Study Approval No. 201 CNU IACUC-YB-R-2015-50).

\section{Bronchoalveolar fluid (BALF) analysis}

BALF analysis was conducted as previously described [28]. The animal study was conducted twice. The first study group consisted of seven animals, with BALF analyzed in three mice, and the remaining mice $(n=4)$ used for morphological studies such as hematoxylin and eosin (H\&E) staining and periodic acid-Schiff (PAS) staining. Further analyses included immunohistochemistry, realtime polymerase chain reaction (RT-PCR), and ELISAs. To confirm the results, a second animal study was conducted. One day after the final treatment, the mice were anesthetized with intraperitoneal injections of $50 \mathrm{mg} / \mathrm{kg}$ Zoletil (Virbac, Carros, France), and the tracheas were cannulated with disposable animal feeding needles. Lavages were performed with three $0.4 \mathrm{~mL}$ aliquots of cold phosphate-buffered saline (PBS). BALF samples were collected and immediately centrifuged at $3000 \mathrm{rpm}$ for 5 min (Sorvall Legend Micro 17R, Thermo Fisher Scientific, Inc. Waltham, MA, USA). The cell pellets were resuspended in PBS for total and differential cell 
counts. The number of total cells and differential cells was counted with a Hemavet Multi-Species Hematology System (Drew Scientific Inc., Waterbury, CT, USA). Some animals after collecting cells and the others which were not used for cell collection were sacrificed with additional Zoletil injection. IgE levels in the serum were measured using a specific mouse IgE ELISA kit (BD Bioscience, catalog number 555248, San Jose, CA, USA) according to the manufacturer's protocols.

\section{Histopathological analysis}

Histopathological analysis was conducted as previously described [28]. Lung tissues were fixed in $10 \%(\mathrm{v} / \mathrm{v})$ formaldehyde solution, dehydrated in a graded ethanol series (99.9\%, 90\%, 80\%, and 70\%), and embedded in paraffin. A total of eight animals from two studies were used, with eight animals per group used for histological analysis. Lung tissues were sectioned $(4 \mu \mathrm{m})$ longitudinally and stained with H\&E and PAS. Eight mice per each group were used for quantitative analysis and after the slides were analyzed with image analyzing software (NIS-Elements BR 4.50.00) all of them were blindly read by the two pathologists. At the first time one of them read the representative slide and finally the other evaluated all of them. Quantitative analysis of the morphological changes, such as goblet cell hyperplasia and inflammatory cell infiltration, was conducted using the $\mathrm{H} \& \mathrm{E}$ stained tissue. Mucous hypersecretion and relative basement membrane (BM) thickening were determined with the PAS stained tissue. Goblet cell hyperplasia, inflammatory cell infiltration, and mucous hypersecretion scores were evaluated from 0 (none) to 5 (severe). The score of relative BM thickening was calculated as follows:

(1) $\mathrm{BM}$ thickness = area of BM/length of $\mathrm{BM}$

(2) Relative BM thickening = BM of each treatment group/BM of the control group

\section{RT-PCR}

To evaluate changes in cDNA levels of IFN- $\gamma$, IL-12p40, IL4, IL-13, TNF- $\alpha$, and IL-6, which are related to asthma induction, RT-PCR analysis was conducted as suggested by Bustin et al. [29]. Total RNA was extracted from the lung using the RNeasy Mini Kit (Qiagen, Hilden, Germany) according to the manufacturer's instructions. Total RNA (100 ng) was used as a template for the reaction. Primers were synthesized for RT-PCR as follows: IFN- $\gamma$ forward $5^{\prime}$ GGCCATCAGCAACAACATAAG-3', IFN- $\gamma$ reverse $5^{\prime}$ GTTGACCTCAAACTTGGCAATAC-3'; IL-12p40 forward 5'-GGACCAAAGGGACTATGAGAAG-3', IL12p40 reverse 5'-CTTCCAACGCCAGTTCAATG-3'; IL4 forward 5'-ACAGGAGAAGGGACGCCAT-3', IL-4 reverse 5'-GAAGCCCTACAGACGAGCTCA-3'; IL-13 forward 5'-CAGCCCTCAGCCATGAAATA-3', IL-13 reverse 5'-CTTGAGTGTGTAACAGGCCATTCT-3'; IL6 forward 5'-GATAAGCTGGAGTCACAGAAG G-3', IL-6 reverse 5'-TTGCCGAGTAGATCTCAAAGT G-3'; TNF- $\alpha$ forward 5'-CTGAGTTCTGCAAAGGGAGA G-3', TNF- $\alpha$ reverse 5'-CCTCAGGGAAGAATCTG GAAAG-3'; GAPDH forward 5'-GTGGAGTCAT ACTGAACATGTAG-3', GAPDH reverse 5'AATGGTGAAGGTCGGTGTG-3'. The RT-PCR cycles consisted of denaturation at $95^{\circ} \mathrm{C}$ for $5 \mathrm{~s}$, and annealing/ extension at $65^{\circ} \mathrm{C}$ for $30 \mathrm{~s}$ for 40 cycles.

\section{ELISA}

To analyze the levels of IFN- $\gamma$, IL-12p40, IL-4, IL-6, and TNF- $\alpha$ in lung tissue, OptEIA mouse ELISAs were purchased from BD Biosciences. IL-13 levels were assessed using the AbFrontier Cymax mouse ELISA kit (AbFrontier, Seoul, Korea). All assays were conducted according to the manufacturers' guidelines. All lung samples were prepared by lysis buffer made with a protease inhibitor cocktail and RIPA buffer (Thermo Fisher Scientific). Aliquots of lung tissue from all groups were weighed and homogenized with lysis buffer. They were centrifuged at $8200 \mathrm{rpm}$ for $15 \mathrm{~min}$, and the supernatants were harvested and measured using a microplate reader (EZ Read 400, Biochrom, Cambourne, UK).

\section{IHC analysis}

IHC analysis was conducted as previously described [28]. Deparaffinized tissue sections were treated with $3 \%$ hydrogen peroxide in methanol for $10 \mathrm{~min}$ to remove endogenous peroxidase. Antigen retrieval was carried out with sodium citrate buffer $(0.1 \mathrm{M})$ using the microwave method. The slides were incubated with normal serum to block nonspecific binding and then incubated for $1 \mathrm{~h}$ with primary antibodies (diluted 1:100 to 1:200) such as IFN- $\gamma$ (sc-74104, Santa Cruz Biotechnology, Dallas, TX, USA), IL-12p40 (sc-57258), IL-4 (sc-73318), IL-13 (sc-1776), TNF- $\alpha$ (MyBioSource, San Diego, CA, USA), and IL-6 (Novus Biologicals, Littleton, CO, USA). The slides were incubated for $10 \mathrm{~min}$ with biotinylated secondary antibodies (Vector Laboratories, PK-7800, Burlingame, CA, USA) and horseradish peroxidase-conjugated streptavidin. Signals were detected using the 3,3-diaminobenzidine tetrahydrochloride substrate chromogen solution, and the cells were counterstained with Mayer's hematoxylin. 


\section{Constituent profiling by high-performance liquid chromatography (HPLC)}

All analyses were performed using an Alliance 2695 HPLC system (Waters; Milford, MA, USA) equipped with a photodiode array detector. The analytical column was an Agilent Zorbax Extend-C18 (5 $\mu \mathrm{m}$, $150 \mathrm{~mm} \times 5 \mathrm{~mm}$ ) with a mobile phase consisting of solvent A (acetonitrile) and solvent B (water containing $0.2 \%$ phosphoric acid) employing gradient elution (from $10 / 90$ to $100 / 0, \mathrm{v} / \mathrm{v}$ ) at a flow rate of $0.5 \mathrm{~mL} /$ min (Table 1). The column temperature was maintained at $25^{\circ} \mathrm{C}$, and the detection wavelength was set at $270 \mathrm{~nm}$ for rutin and quercetin. The solvent was filtered through a $0.22-\mu \mathrm{m}$ filter and degassed. The sample injection volume was $10 \mu \mathrm{L}$.

\section{Statistical analysis}

Results are expressed as means \pm standard deviation (SD). Group differences were evaluated by one-way analysis of variance, followed by Dunnett's multiple comparisons test. A $p$ value of $<0.01$ or $<0.05$ was considered statistically significant.

\section{Results}

\section{O. humifusa extract has $3.9 \%$ pharmaceutical active compounds}

The nutritional composition of the extract is shown in Table 2 . The extract contains $24 \%$ of the recommended daily allowance (RDA) of carbohydrate $(77.8 \mathrm{~g} / 100 \mathrm{~g})$, $9 \%$ of the RDA of protein $(11.5 \mathrm{~g} / 100 \mathrm{~g}), 2 \%$ of the RDA of fat $(4.9 \mathrm{~g} / 100 \mathrm{~g})$, and $1 \%$ of the RDA of cholesterol $(1.9 \mathrm{~g} / 100 \mathrm{~g})$.

The total percentage of carbohydrate, protein, and fat in the extracts was $96.1 \%$, and the chemical substances that contain pharmaceutically active compounds were estimated to be $3.9 \%$. The extracts contained the active compound such as rutin $(0.38 \%)$ and quercetin (0.1\%). The two active compounds
Table 2. Analysis of nutritional components in the 0 . humifusa extract.

\begin{tabular}{lcr}
\hline Components & Values (units/100 g) & RDA (\%) $)^{\mathrm{a}}$ \\
\hline Energy (kcal) & 342.5 & \\
Carbohydrate (g) & 77.8 & 330 \\
Protein (g) & 11.5 & 55 \\
Fat $(\mathrm{g})$ & 4.9 & 50 \\
Cholesterol (mg) & 1.9 & 300 \\
\hline
\end{tabular}

${ }_{\mathrm{a} D A}$, recommended daily allowance.

accounted for about $12.3 \%$ of the total chemical composition and are thought to have anti-asthmatic efficacy along with undetermined components.

\section{O. humifusa inhibited white blood cell proliferation and suppressed neutrophil levels}

The number of white blood cells (WBC) was significantly higher (Figure 1(a)) in the OVA-induced asthmatic group than that in the control group in which the vehicle was orally administered and not inhaled. Dexamethasone, which is commonly used as an asthma drug, suppressed OVA-induced increases in WBCs. Similarly, O. humifusa dose-dependently modulated OVA-induced increases in WBCs. Increased eosinophils in the bronchoalveolar fluid (BALF) is commonly observed in asthma patients; however, an increase in neutrophils is occasionally observed [30]. OVA increased the number of neutrophils, whereas they were suppressed by dexamethasone (Figure 1(b)); $O$. humifusa suppressed the level of neutrophils in a dosedependent manner. Neutrophil counts of the $500 \mathrm{mg} /$ $\mathrm{kg} O$. humifusa-treated group were similar to those of the dexamethasone-treated group. Likewise, changes in IgE levels were similar to the observed changes in WBC count (Figure $1(\mathrm{a}, \underline{\mathrm{c}})$ ). OVA-induced increases in $\operatorname{IgE}$ were suppressed by dexamethasone and O. humifusa treatment. Specifically, $500 \mathrm{mg} / \mathrm{kg}$ O. humifusa treatment significantly suppressed IgE levels; there were nonsignificant decreases in IgE levels in OVA-sensitized mice treated with $250 \mathrm{mg} / \mathrm{kg}$ O. humifusa and $50 \mathrm{mg} / \mathrm{kg}$ O. humifusa.

Table 1. Analytical conditions of HPLC for analysis of 0 . humifusa.

\begin{tabular}{|c|c|c|c|}
\hline Parameters & \multicolumn{3}{|c|}{ Conditions } \\
\hline $\begin{array}{l}\text { Column } \\
\text { Flow rate } \\
\text { Injection Volume } \\
\text { UV detection } \\
\text { Run time }\end{array}$ & & $\begin{array}{r}\text { ended-C18 (C18, } \\
1 \mathrm{~mL} / \\
10 \\
270 \\
30 \mathrm{~m} \\
\end{array}$ & $5 \mu \mathrm{m})$ \\
\hline & Time (min) & Acetonitrile (\%) & $0.2 \%$ phosphoric acid (\%) \\
\hline Gradient & $\begin{array}{r}0 \\
7 \\
18 \\
19 \\
30\end{array}$ & $\begin{array}{r}10 \\
10 \\
100 \\
10 \\
10\end{array}$ & $\begin{array}{r}90 \\
90 \\
0 \\
90 \\
90\end{array}$ \\
\hline
\end{tabular}



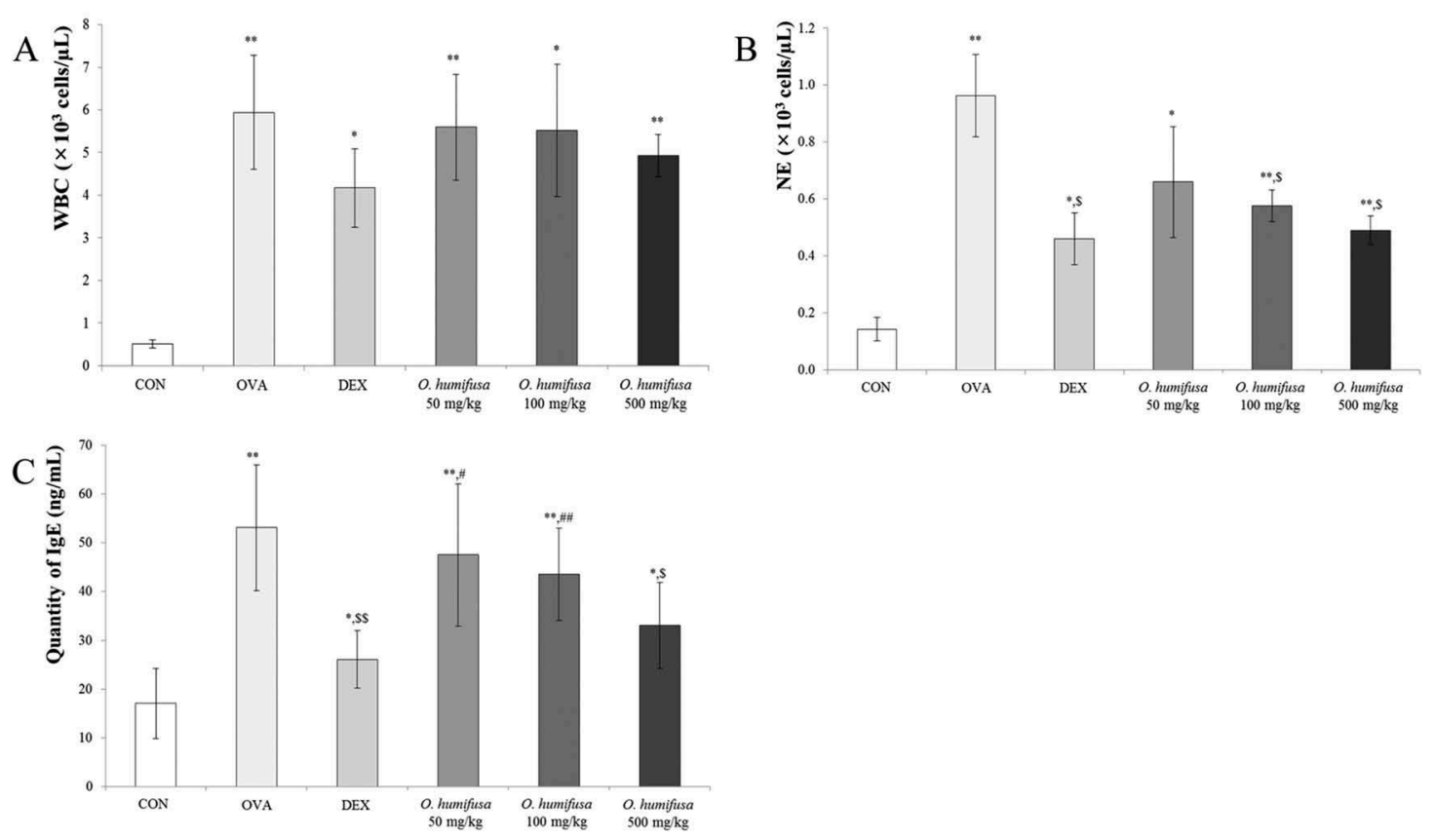

Figure 1. O. humifusa decreased the number of white blood cells (WBCs) and dose-dependently suppressed neutrophils and lgE in bronchoalveolar lavage fluid (BALF) from ovalbumin (OVA)-induced asthma in mice. (a) Although the differences were not significant, $O$. humifusa decreased WBC counts in BALF. (b) O. humifusa dose-dependently suppressed neutrophils in BALF following OVA treatment. (c) Similar to neutrophils, O. humifusa dose-dependently attenuated OVA-induced increases in IgE levels. Each bar represents the means $\pm \mathrm{SD}(N=6) .{ }^{*} p<0.05$ vs. control; ${ }^{* *} p<0.001$ vs. control; ${ }^{\$} p<0.05$ vs. asthma induction; ${ }^{\$ \$} p<0.01$ vs. asthma induction; ${ }^{\#} p<0.05$ vs. dexamethasone; ${ }^{\# \#} p<0.01$ vs. dexamethasone.

\section{O. humifusa significantly suppressed morphological changes in OVA-induced asthma}

OVA treatment caused morphological changes in lung tissue that were typical of asthma, including inflammatory cell infiltration near bronchioles or vessels (arrows in Figure 2(Ab)), mucous hypersecretion ( $M$ in Figure $2(\mathrm{Ab}, \mathrm{Bb}))$, and bronchial epithelial cell hyperplasia (Figure 2(Aa,Ba)). Dexamethasone restored OVA-induced lung damage (Figure $2(\mathrm{Ac}, 2 \mathrm{Bc})$ ) to that observed in the lungs with normal morphology. We further confirmed the changes in OVA-induced lung damage after treatment with dexamethasone or $O$. humifusa treatment by quantitative assessments (Table 3). Goblet cell hyperplasia was only observed in the OVA- and $50-100 \mathrm{mg} / \mathrm{kg}$ O. humifusa-treated groups. The inflammatory cell infiltration score for the OVA-treated group was 2, whereas the scores of other treatment groups decreased to 1 . Inflammatory cell infiltration was not observed in the control or dexamethasone-treated groups. The inflammatory cell infiltration score reached as high as 5 in the OVAsensitized mice, an effect that was dose-dependently decreased by $O$. humifusa treatment. Specifically, mice treated with 50,100 , and $500 \mathrm{mg} / \mathrm{kg} \mathrm{O}$. humifusa had a score of 4, 3, and 2, respectively. Mucous hypersecretion was defined in the same manner as that of goblet cell hyperplasia or inflammatory cell infiltration (from 0 (none) to 5 (severe)). Mucous hypersecretion was observed only in the OVA and $50 \mathrm{~g} / \mathrm{kg}$ O. humifusa treatment groups. These results suggest that OVAinduced mucous hypersecretion is suppressed by dexamethasone or O. humifusa treatment at doses greater than $100 \mathrm{mg} / \mathrm{kg}$. The results of experiments measuring relative $\mathrm{BM}$ thickening were very similar to those of the inflammatory cell infiltration experiments; ovalbumin treatment induced relative thickening of the $\mathrm{BM}$, an effect that was dose-dependently attenuated by $O$. humifusa.

\section{0. humifusa decreased Th1-related cytokines such as IFN- $\gamma$ and IL-12}

To evaluate changes in Th1-related cytokines such as IFN- $\gamma$ and IL-12, we measured gene expression levels by RT-PCR and proteins levels by enzyme-linked immunoassay (ELISA) and immunohistochemistry (IHC). OVA upregulated IL-12 gene expression (Figure 3(a)). Although there were no statistical 


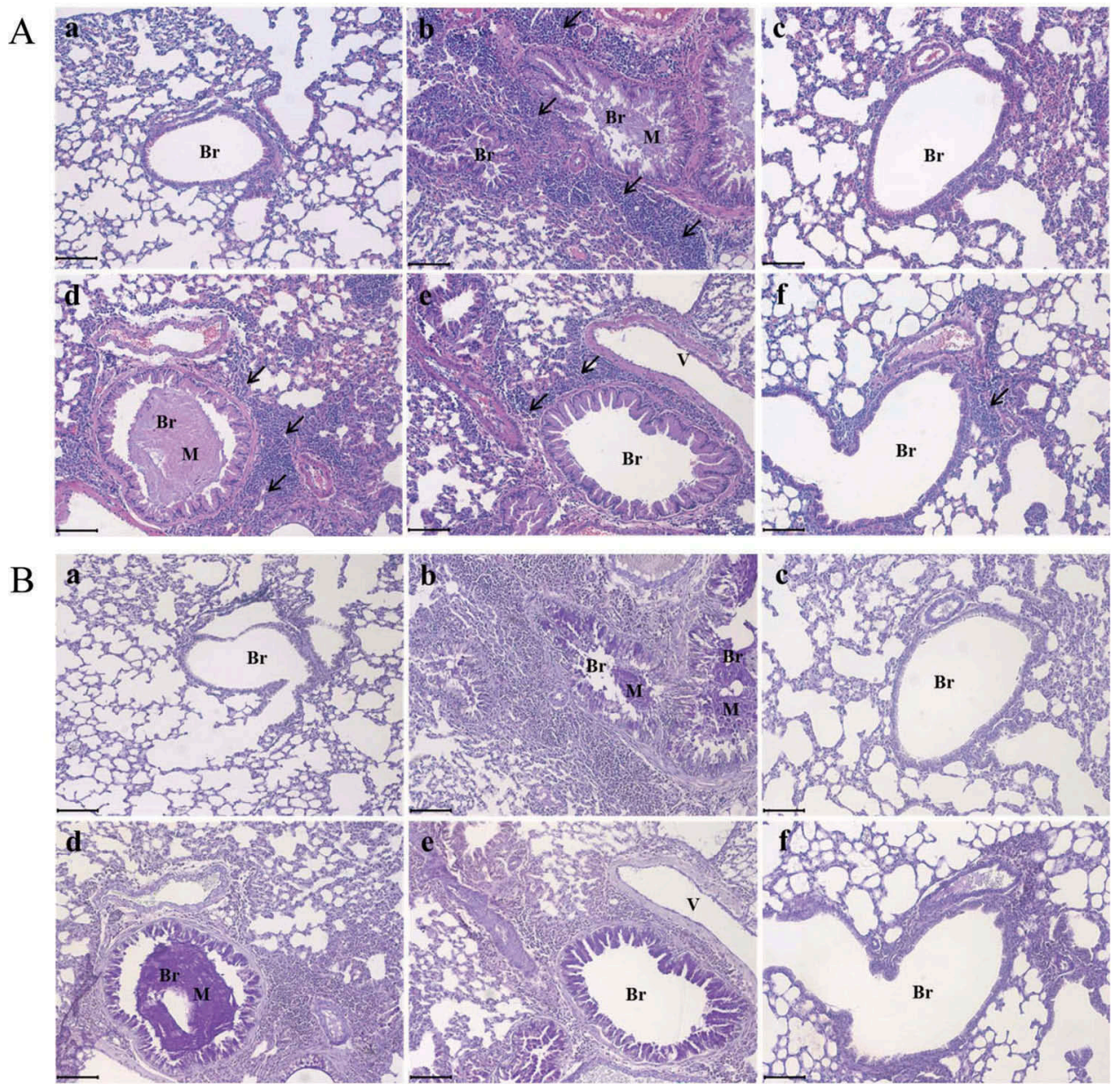

Figure 2. O. humifusa dose-dependently suppressed typical morphological changes induced by OVA treatment in the respiratory system. (a) In the H\&E-stained lung, O. humifusa significantly modulated asthmatic changes, such as mucous hypersecretion (M), inflammatory cell infiltration in the intercellular space (arrows), epithelial cell hyperplasia, and airway remodeling. (b) In the PAS stained lung, $O$. humifusa prevented mucous secretion in a dose-dependent manner. $N=8$. Scale Bar: $100 \mu \mathrm{m}$. Br, bronchiole; $\mathrm{V}$, vessel; $M$, mucous; arrow, inflammatory cell; a, vehicle control; b, asthma induction; c, dexamethasone; d, $50 \mathrm{mg} / \mathrm{kg} / \mathrm{day} 0$. humifusa; e, $100 \mathrm{mg} / \mathrm{kg} /$ day O. humifusa; $\mathrm{f}, 500 \mathrm{mg} / \mathrm{kg} /$ day 0 . humifusa.

Table 3. Quantitative results of histopatholgoical changes.

\begin{tabular}{|c|c|c|c|c|}
\hline & $\begin{array}{l}\text { Goblet cell hyperplasia } \\
(0-5)\end{array}$ & $\begin{array}{l}\text { Inflammatory cell infiltration } \\
(0-5)\end{array}$ & $\begin{array}{c}\text { Mucous hypersecretion } \\
(0-5)\end{array}$ & $\begin{array}{c}\text { Relative basement membrane } \\
\text { thickening }\end{array}$ \\
\hline CON & $0 \pm 0.3$ & $0 \pm 0.5$ & $0 \pm 0.4$ & $1.00 \pm 0.05$ \\
\hline OVA & $2 \pm 0.5^{*}$ & $5 \pm 0.9^{* *}$ & $5 \pm 0.6^{* *}$ & $1.11 \pm 0.08^{*}$ \\
\hline DEX & $0 \pm 0.5^{\$}$ & $0 \pm 0.5^{\$ \$}$ & $0 \pm 0.5^{5 \$}$ & $0.82 \pm 0.09^{\$}$ \\
\hline O. humifusa 50 mg/kg & $1 \pm 0.4^{*}$ & $4 \pm 0.8^{* * \#}$ & $5 \pm 0.8^{* * \# \#}$ & $0.94 \pm 0.04$ \\
\hline O. humifusa $100 \mathrm{mg} / \mathrm{kg}$ & $1 \pm 0.5 *$, & $3 \pm 0.4^{*}$ & $0 \pm 0.3^{\$ \$^{\prime}}$ & $0.90 \pm 0.07^{\$}$ \\
\hline O. humifusa $500 \mathrm{mg} / \mathrm{kg}$ & $0 \pm 0.4^{s^{\prime}}$ & $2 \pm 0.8^{*}{ }^{\prime}, \#$ & $0 \pm 0.5^{\$ \$}$ & $0.88 \pm 0.04^{\$}$ \\
\hline
\end{tabular}

Each bar represents the means \pm SD $(N=8)$.

${ }^{*} p<0.05$ vs. control; ${ }^{* *} p<0.001$ vs. control; ${ }^{\$} p<0.05$ vs. asthma induction; ${ }^{\$ \$} p<0.01$ vs. asthma induction; ${ }^{\#} p<0.05$ vs. dexamethasone; ${ }^{\# \#} p<0.01$ vs. dexamethasone.

differences, O. humifusa slightly suppressed the levels of IL-12 (Figure $3(\mathrm{~b}, \mathrm{c})$ ). IFN- $\gamma$ gene expression was upregulated by OVA treatment; however, dexamethasone and $O$. humifusa suppressed IFN- $\gamma$ gene expression at all concentrations tested (Figure 3(a)). IFN- $\gamma$ protein levels were dose-dependently modulated by $O$. humifusa (Figure 3(b,d)).
O. humifusa dose-dependently suppressed the levels of the Th2-related cytokines IL-4 and IL-13, but not IL-5

Expression of both IL-4 and IL-13 genes was dose-dependently downregulated by $O$. humifusa treatment, whereas their expression was upregulated after treatment with 

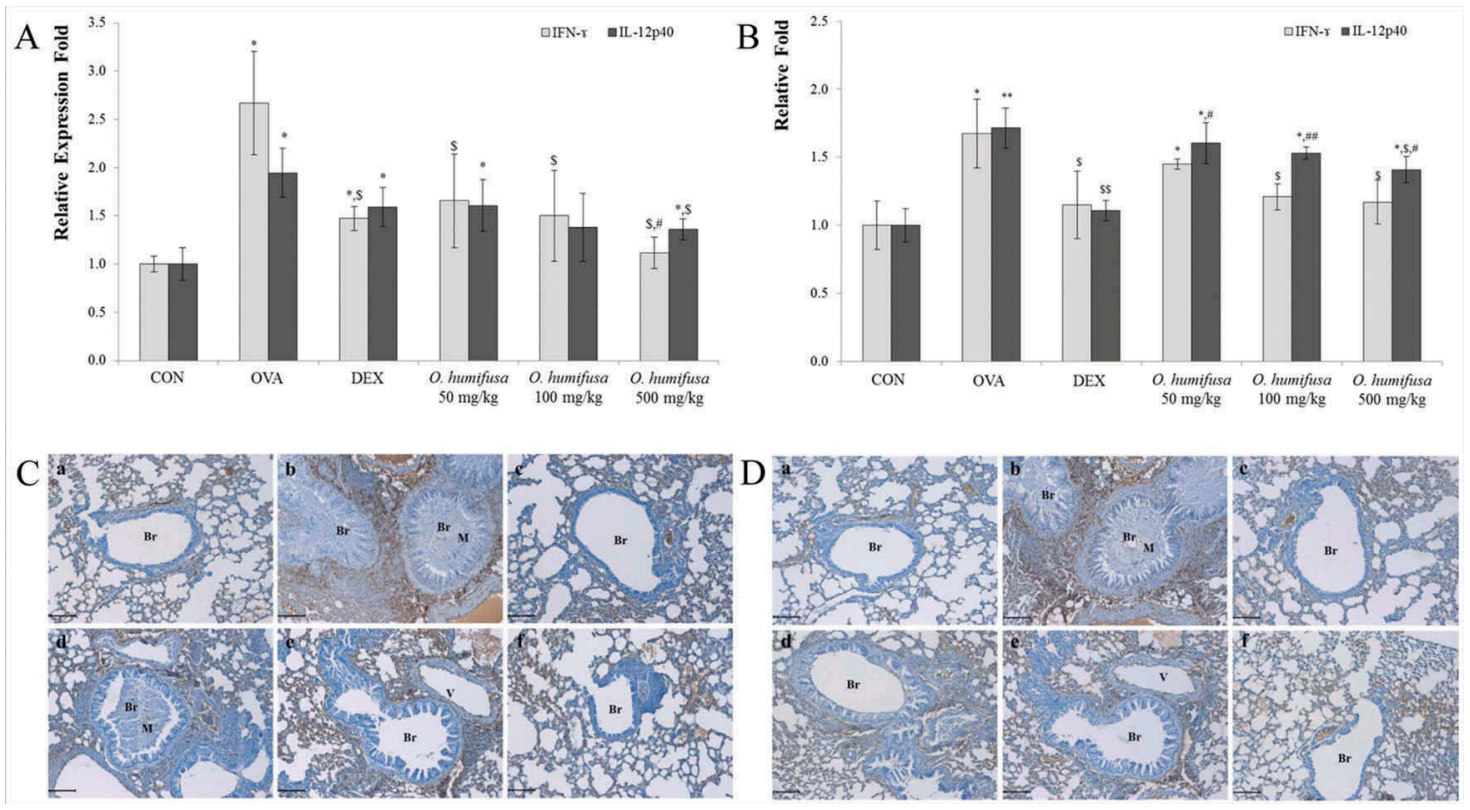

Figure 3. 0 . humifusa modulated gene expression and protein levels of Th1-related cytokines such as IFN- $\gamma$ and IL-12p40. 0 . humifusa suppressed not only IFN- $\gamma$ gene expression as quantified by RT-PCR (A), but also IFN- $\gamma$ protein levels, as assessed by ELISA (B) and IHC (C). O. humifusa modulated IL-12 gene expression, as confirmed by RT-PCR (A) and significantly inhibited the protein expression of IL-12p40, as verified by ELISA (B) and IHC (D). $N=8$. Scale Bar: $100 \mu \mathrm{m}$. Br, bronchiole; V, vessel; $M$, mucous; a, vehicle control; b, asthma induction; c, dexamethasone; d, $50 \mathrm{mg} / \mathrm{kg} /$ day 0 . humifusa; e, $100 \mathrm{mg} / \mathrm{kg} /$ day 0. humifusa; f, $500 \mathrm{mg} / \mathrm{kg} / \mathrm{day} 0$. humifusa. ${ }^{*} p<0.05$ vs. control; ${ }^{* *} p<0.001$ vs. control; ${ }^{\$} p<0.05$ vs. asthma induction; ${ }^{\$ \$} p<0.01$ vs. asthma induction; ${ }^{\#} p<0.05$ vs. dexamethasone; ${ }^{\# \#} p<0.01$ vs. dexamethasone.

ovalbumin (Figure 4(a)). Expression profiles in the $500 \mathrm{mg} / \mathrm{kg}$ O. humifusa treatment group were lower than those in the dexamethasone treatment group; changes in IL-4 and IL-13 protein levels followed a similar pattern (Figure 4(b-d)). Protein levels increased following dexamethasone treatment and decreased in a dose-dependent manner following O. humifusa treatment. IL-4 and IL-14 gene expression and protein levels were significantly regulated by $O$. humifusa treatments. Therefore, IL-4 and IL-13 may be key factors in suppressing the induction of asthma. IL-5, however, they were not modulated by $O$. humifusa treatment (data not shown). IL-4 and IL-13 promote IgE production [31]; changes in IgE levels (Figure 1(c)) reflected those of IL4 and IL-13 (Figure 4).

\section{O. humifusa dose-dependently decreased the Th17-related cytokines TNF- $a$ and IL-6}

TNF- $\alpha$ gene expression was upregulated by OVA treatment, an effect that was reversed by dexamethasone treatment to levels observed in the control group. $O$. humifusa treatment downregulated TNF-a gene expression in a dose-dependent manner (Figure 5(a)); a similar pattern was observed for the IL-6 gene. Expression of TNF- $\alpha$ and IL- 6 genes after treatment with $500 \mathrm{mg} / \mathrm{kg}$ O. humifusa was similar to that of the dexamethasone treatment group. Although O. humifusa suppressed both TNF- $\alpha$ and IL- 6 gene expression, it was more effective at suppressing IL-6 expression (Figure 5(b,d)). TNF- $\alpha$ and IL-6 were dose-dependently downregulated by O. humifusa treatment; however, the expression of IL-6 was similar to that observed in the dexamethasone treatment group after treatment with 100 and $500 \mathrm{mg} / \mathrm{kg}$ O. humifusa.

\section{Discussion}

Asthma is initially induced by antigen-presenting cells (APCs) that recognize a repeated allergen such as pollen, pet dander, dust mites, tobacco smoke, and environmental pollutants. For several decades, studies have attempted to elucidate the relationship between $\operatorname{IgE}$ and type 1 allergies, which are representative of asthma etiology [31,32]. The results of the present study suggest that reduced IgE expression regulates ovalbumininduced asthma. Further, the balance between Th1 and Th2 activity is important for maintaining immunological homeostasis; an imbalance may result in the induction of various diseases, including asthma [33]. In asthmatic patients [1], Th2 cells recognize APCs and release cytokines such as IL-4 and IL-13, which stimulate class switching in B cells that produce $\operatorname{IgE}$ [31]. Changes in IFN- $\gamma$ levels are related to IgE modulation 

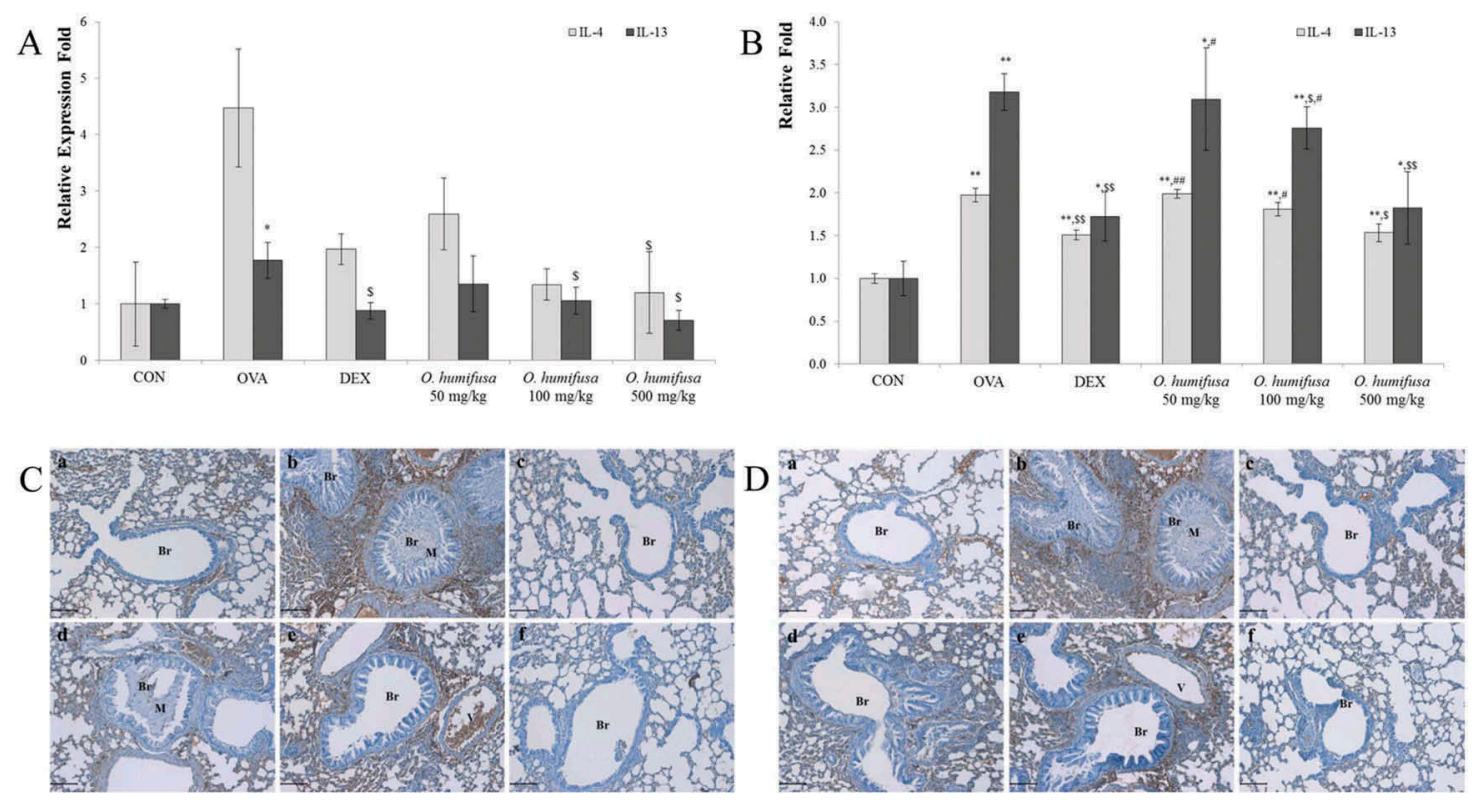

Figure 4. 0 . humifusa significantly suppressed Th2-related cytokines such as IL-4 and IL-13. Although 0 . humifusa downregulated gene expression levels of IL-4, we found no statistical differences when using RT-PCR (A); O. humifusa significantly suppressed IL-4 gene expression as assessed by ELISA (B) and IHC (C). Similarly, IL-13 gene expression levels (A) and protein levels (B, D) were dosedependently decreased by $O$. humifusa treatments. $\mathrm{N}=8$. Scale bar: $100 \mu \mathrm{m}$. Br, bronchiole; $\mathrm{V}$, vessel; $\mathrm{M}$, mucous; a, vehicle control; $\mathrm{b}$, asthma induction; c, dexamethasone; d, $50 \mathrm{mg} / \mathrm{kg} /$ day 0 . humifusa; e, $100 \mathrm{mg} / \mathrm{kg} /$ day 0 . humifusa; $\mathrm{f}, 500 \mathrm{mg} / \mathrm{kg} /$ day 0 . humifusa. ${ }^{*} p<0.05$ vs. control; ${ }^{* *} p<0.001$ vs. control; ${ }^{\$} p<0.05$ vs. asthma induction; ${ }^{\$ \$} p<0.01$ vs. asthma induction; ${ }^{\#} p<0.05$ vs. dexamethasone; ${ }^{\# \#} p<0.01$ vs. dexamethasone.
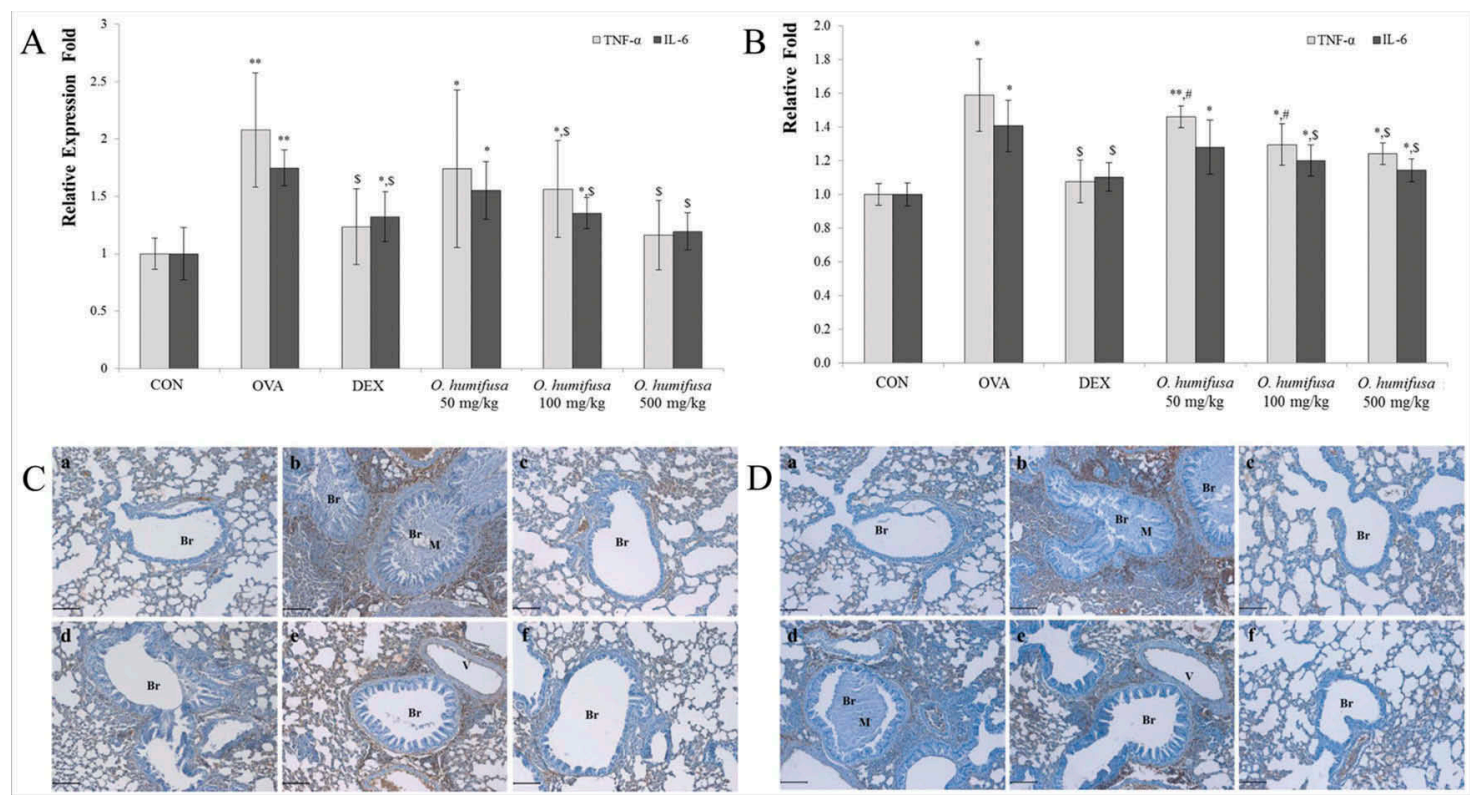

Figure 5. 0 . humifusa reduced gene expression and protein levels of Th17-related cytokines such as TNF-a and IL-6. 0 . humifusa down-regulated the expression of TNF- $a$ and IL-6, as confirmed by RT-PCR (A), and their protein levels as assessed by ELISA (B) and IHC (C) in lung tissues; several were reduced in a dose-dependent manner. $\mathrm{N}=8$. Scale bar: $100 \mu \mathrm{m}$. Br, bronchiole; $\mathrm{V}$, vessel; $\mathrm{M}$, mucous; a, vehicle control; b, asthma induction; c, dexamethasone; $d, 50 \mathrm{mg} / \mathrm{kg} /$ day 0 . humifusa; e, $100 \mathrm{mg} / \mathrm{kg} /$ day $O$. humifusa; $f$, $500 \mathrm{mg} / \mathrm{kg} /$ day 0 . humifusa. ${ }^{*} p<0.05$ vs. control; ${ }^{* *} p<0.001$ vs. control; ${ }^{\$} p<0.05$ vs. asthma induction; ${ }^{\#} p<0.05$ vs. dexamethasone. 
[34]; under most known conditions of asthma induction, IgE levels increase [8].

In asthma, Th2-related cytokines such as IL-4 [3537], IL-5 [38], and IL-13 [35,37] significantly increase; the development of antibodies against Th2-related cytokines is an active field of research [39]. Changes in Th17 cells correlate with asthma induction, making it important to analyze Th-17 proteins such as TNF- $\alpha$, IL-6, and IL-1 $\beta$ [40]. We previously reported a correlation between TNF- $\alpha$ gene expression [41] and protein levels [42] and the induction of asthma. TNF- $\alpha$ has a chemoattractant effect on neutrophils and eosinophils [17]. Although an increase in eosinophil levels is observed in many asthma patients, other patients show a surge in neutrophils [30]. As shown in Figure 1(b), O. humifusa may regulate neutrophil levels, which are related to TNF- $\alpha$ functions (Figure 5(a,b,d)).

Both IL-6 and IL-13 levels are usually elevated in asthma patients and are presumed to be closely correlated [19]; we found that IL-6 levels were similar to IL13 levels, supporting this idea. IL-13 and IL-6 levels after treatment with $500 \mathrm{mg} / \mathrm{kg}$ O. humifusa were notably lower than their levels after dexamethasone treatment.

To our knowledge, this is the first study to report the identification of active compounds from the $O$. humifusa leaf. We identified active compounds related to anti-asthma effects in the O. humifusa leaf using HPLC analysis, including bioactive markers related to anti-asthmatic activity such as rutin $(0.38 \%, \mathrm{w} / \mathrm{w})$ and quercetin $(0.1 \%, \mathrm{w} / \mathrm{w})$ (Figure 6$)$.

We identified rutin and quercetin as two bioactive compounds found in the O. humifusa leaf. Cho et al. suggested the possibility of anti-inflammatory effect of ethylacetate fraction from $O$. humifusa using macrophage cells such as Raw 264.7 and identified the quercetin as one of the key components in O. humifusa [43]. Karym et al. reported that Opuntia humifusa-indica contains rutin in the range of $0.002-0.02 \%(\mathrm{w} / \mathrm{w})$. Similary, we identified rutin in $\mathrm{O}$. humifusa $(0.38 \%)$, and rutin content in $O$. humifusa was 19 times higher than that of O. ficus-indica [44]. Cho et al. reported that $O$. humifusa leaf has an antiinflammatory effect, and quercetin was reported as one of the key marker. In the present study, we have reported that O. humifusa leaf has anti-asthmatic activity and rutin and quercetin as biomarkers. Rutin and quercetin have been reported to contribute to anti-inflammatory efficacy as well as anti-asthmatic efficacy. Jung et al. [45] studied the effects of rutin and quercetin on asthmatic responses in OVA-sensitized, conscious guinea pigs. Rutin and quercetin $(7.5 \mathrm{mg} / \mathrm{kg}$, oral administration) notably and dose-dependently inhibited airway resistance in both immediate-phase and late-phase responses. At a dose of $15 \mathrm{mg} / \mathrm{kg}$, rutin and quercetin also inhibited production of histamine, phospholipase 2, and erythropoietin. Thus, rutin and quercetin may be beneficial compounds for treating asthma. Therefore, if the effective dosage for rutin and quercetin in guinea pigs is $7.5 \mathrm{mg} / \mathrm{kg} /$ day, the human equivalent dosage is $97 \mathrm{mg} / 60 \mathrm{~kg} /$ day (per os). Extracts of $O$. humifusa showed anti-asthmatic effects at two evaluated doses and significantly suppressed the production of IL-4 and IL-13 at a dose of $500 \mathrm{mg} / \mathrm{kg}$. We calculated the optimal oral intake of O. humifusa extract using the conversion factor between human and mouse (12.33). Therefore, if the effective dosage for mice is $500 \mathrm{mg} / \mathrm{kg} /$ day, the human equivalent dosage is $2425 \mathrm{mg} / 60 \mathrm{~kg} /$ day as extract or 10 and $2.5 \mathrm{mg} / 60 \mathrm{~kg} /$ day for rutin and quercetin, respectively. Other pharmaceutically active compounds other than rutin and quercetin may also be involved in the anti-asthmatic effects of $O$. humifusa and may have synergistic effects. Although

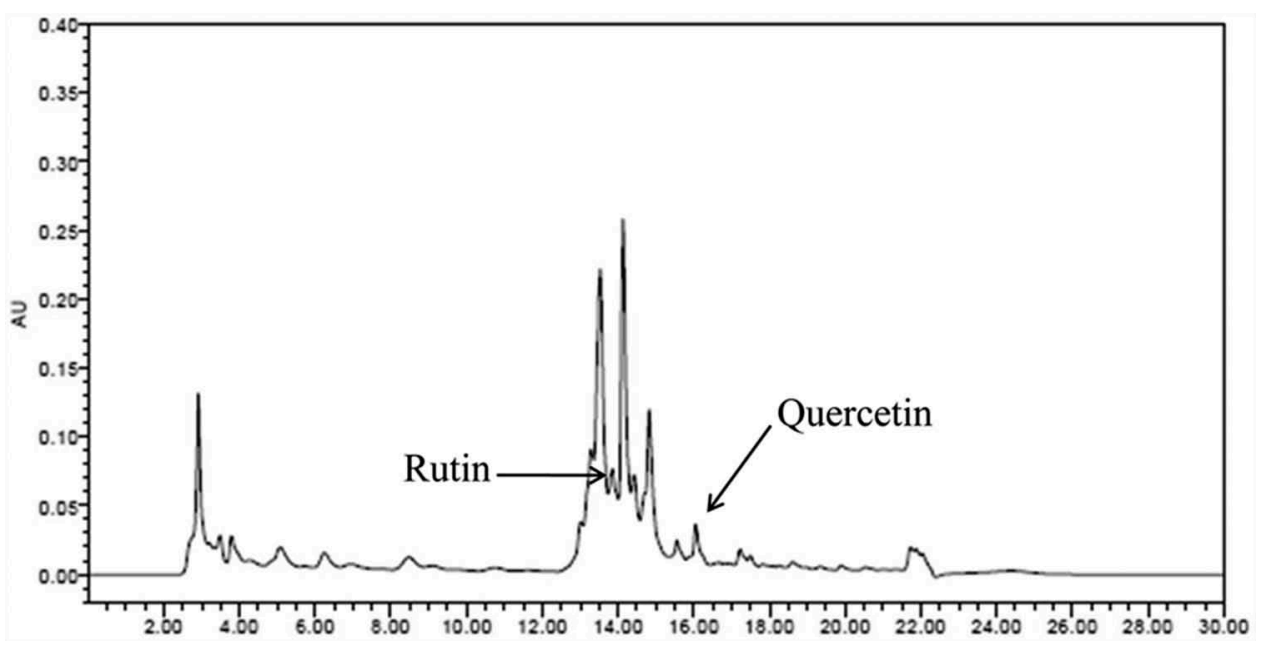

Figure 6. HPLC analysis of 0 . humifusa. We identified rutin $(0.38 \pm 0.005 \%$ at 13.85 min retention time $)$ and quercetin $(0.1 \pm 0.001 \%$ at 16.05 min retention time) as components of 0 . humifusa. 
O. humifusa has been studied for its biological effects in the treatment of diseases such as pancreatitis, cancer, and diabetes, nothing is known about the anti-asthmatic constituents of $O$. humifusa $[24,46,47]$. In the present study, we report anti-asthmatic effects and related constituents of O. humifusa for the first time. Further studies are needed to better understand the active constituents and pharmacological properties of O. humifusa.

O. humifusa effectively suppressed OVA-induced asthma by modulating Th1-/Th2-/Th17-related cytokines; in particular, Th2-related cytokines such as IL-4 and IL-13 were downregulated. O. humifusa prevented pulmonary morphological changes such as mucous hypersecretion, epithelial hyperplasia, and inflammatory cell infiltration near bronchioles and vessels. Although there are biochemical constituents in O. humifusa, the anti-asthmatic effects may be derived from rutin and quercetin. We conclude that $O$. humifusa is a potential functional food.

\section{Author contributions}

S-Y Lee and C-S Bae contributed equally to this work; they collected test data and drafted the manuscript. Y-H Choi, $\mathrm{N}-\mathrm{S}$ Seo, C-S Na, and JC Yoo conducted the experiments, including O. humifusa preparation and analyses of the raw data. SS Cho and D-H Park designed the study and interpreted the results.

\section{Acknowledgment}

We deeply thank Professor Jae-Hak Park and Professor MinJae Lee as specialists for contributing to this study by reading the histopatholgical results.

\section{Disclosure statement}

No potential conflict of interest was reported by the authors.

\section{Funding}

The authors raised funds.

\section{ORCID}

Seung Sik Cho (D) http://orcid.org/0000-0003-1497-0529

\section{References}

[1] World Health Organization. Asthma fact sheet. $\mathrm{N}^{\mathrm{o}} 307$. 2013 Nov.

[2] Kay AB. Allergy and allergic diseases. First of two parts. N Engl J Med. 2001;344:30-37.

[3] National asthma education and prevention program. Expert panel report: guidelines for the diagnosis and management of asthma update on selected topics 2002. J Allergy Clin Immunol. 2002;110(suppl 5):S141S219.

[4] Kumar RK, Webb CC, Herbert C, et al. Interferongamma as a possible target in chronic asthma. Inflamm Allergy-Drug Targets. 2006;5(4):253-256.

[5] Mattes J, Yang M, Mahalingam S, et al. Intrinsic defect in $\mathrm{T}$ cell production of Interleukin (IL)-13 in the absence of both IL-5 and eotaxin precludes the development of eosinophilia and airways hyperreactivity in experimental asthma. J Exp Med. 2002;195(11):1433-1444.

[6] Manetti R, Parronchi P, Giudizi MG, et al. Natural killer cell stimulatory factor (interleukin 12 (IL-12)) induces T helper type 1 (Th1)-specific immune responses and inhibits the development of IL-4 producing Th cells. J Exp Med. 1993;177:1199-1204.

[7] Allswede DM, Buka SL, Yolken RH, et al. Elevated maternal cytokine levels. Schizophr Res. 2016;172(13):41-45.

[8] Mosmann TR, Coffman RL. TH1 and TH2 cells: different patterns of lymphokine secretion lead to different functional properties. Annu Rev Immunol. 1989;7:145168.

[9] Hershey GK. IL-13 receptors and signaling pathways: an evolving web. J Allergy Clin Immunol. 2003;111:677690.

[10] Rankin JA, Picarella DE, Geba GP, et al. Phenotypic and physiologic characterization of transgenic mice expressing interleukin 4 in the lung: lymphocytic and eosinophilic inflammation without airway hyperreactivity. Proc Natl Acad Sci U S A. 1996;93:7821-7825.

[11] Wills-Karp M, Luyimbazi J, Xu X, et al. Interleukin-13: central mediator of allergic asthma. Science. 1998;282:2258-2261.

[12] Zhu Z, Homer RJ, Wang Z, et al. Pulmonary expression of interleukin-13 causes inflammation, mucus hypersecretion, sub-epithelial fibrosis, physiologic abnormalities, and eotaxin production. J Clin Invest. 1999;103:779-788.

[13] Kriegler M, Perez C, DeFay K, et al. A novel form of $\mathrm{TNF} /$ cachectin is a cell surface cytotoxic transmembrane protein: ramifications for the complex physiology of TNF. Cell. 1988;53:45-53.

[14] Berry M, Brightling C, Pavord I, et al. TNF- $\alpha$ in asthma. Curr Opin Pharmacol. 2007;7:279-282.

[15] Stadnyk AW. Cytokine production by epithelial cells. FASEB J. 1994;8:1041-1047.

[16] Rincon M, Irvin CG. Role of IL-6 in asthma and other inflammatory pulmonary diseases. Int J Biol Sci. 2012;8 (9):1281-1290.

[17] Lukacs NW, Strieter RM, Chensue SW, et al. TNF-alpha mediates recruitment of neutrophils and eosinophils during airway inflammation. J Immunol. 1995; 154:5411-5417.

[18] Scheurich P, Thoma B, Ucer U, et al. Immunoregulatory activity of recombinant human tumor necrosis factor (TNF)-alpha: induction of TNF receptors on human T cells and TNF-alpha-mediated enhancement of $\mathrm{T}$ cell responses. J Immunol. 1987;138:1786-1790.

[19] Neveu WA, Allard JL, Raymond DM, et al. Elevation of IL-6 in the allergic asthmatic airway is independent of inflammation but associates with loss of central airway function. Respir Res. 2010;11(1):28. 
[20] Barnes PJ. Current issues for establishing inhaled corticosteroids as the anti-inflammatory agents of choice in asthma. J Allergy Clin Immunol. 1998;101:S427-S433.

[21] Wise J. Corticosteroids for asthma may suppress growth in children in first year of treatment, researchers say. BMJ. 2014;349:g4623.

[22] Ciriaco M, Ventrice P, Russo G, et al. Corticosteroidrelated central nervous system side effects. J Pharmacol Pharmacotherapeut. 2013;4(suppl 1):s94-s98.

[23] Silva-Hughe AF, Wedge DE, Cantrell CL, et al. Diversity and antifungal activity of the endophytic fungi associated with the native medicinal cactus Opuntia humifusa (Cactaceae) from the United States. Microbiol Res. 2015;175:67-77.

[24] Hahm S-W, Park J, Oh S-Y, et al. Anticancer properties of extracts from Opuntia humifusa against human cervical carcinoma cells. J Med Food. 2015;18(1):31-44.

[25] Lee J-A, Jung B-G, Kim T-H, et al. Dietary feeding of Opuntia humifusa inhibits UVB radiation-induced carcinogenesis by reducing inflammation and proliferation in hairless mouse model. Photochem Photobiol. 2013;89 (5):1208-1215.

[26] Kang J, Lee J, Kwon D, et al. Effect of Opuntia humifusa supplementation and acute exercise on insulin sensitivity and associations with PPAR- $\gamma$ and PGC- $1 \alpha$ protein expression in skeletal muscle of rats. Int J Mol Sci. 2013;14(4):7140-7154.

[27] Kang J, Park J, Choi SH, et al. Opuntia humifusa supplementation increased bone density by regulating parathyroid hormone and osteocalcin in male growing rats. Int J Mol Sci. 2012;13(6):6747-6756.

[28] Bang M-A, Seo J-H, Seo J-W, et al. Bacillus subtilis KCTC 11782BP-produced alginate oligosaccharide effectively suppresses asthma via T-helper cell type 2related cytokines. PLos One. 2015;10(2):e0117524.

[29] Bustin SA, Benes V, Garson JA, et al. The MIQE guidelines: minimum information for publication of quantitative real-time PCR experiments. Clin Chem. 2009;55 (4):611-622.

[30] Monteseirín J. Neutrophils and asthma. J Investig Allergol Clin Immunol. 2009;19(5):340-354.

[31] Platts-Mills TA. The role of immunoglobulin $\mathrm{E}$ in allergy and asthma. Am J Respir Crit Care Med. 2001;164:S1-S5.

[32] Burrows B, Martinez FD, Halonen M, et al. Association of asthma with serum IgE levels and skin-test reactivity to allergens. N Engl J Med. 1989;320:271-277.

[33] Kidd P. Th1/Th2 balance: the hypothesis, its limitations, and implications for health and disease. Altern Med Rev. 2003;8(3):223-246.
[34] Stirling RG, Chung KF. Future treatments of allergic diseases and asthma. Br Med Bull. 2000;56(4):10371053.

[35] Antczak A, Domańska-Senderowska D, Górski P, et al. Analysis of changes in expression of IL-4/IL-13/STAT6 pathway and correlation with the selected clinical parameters in patients with atopic asthma. Int J Immunopathol Pharmacol. 2016;29(2):195-204.

[36] Wenzel S, Wilbraham D, Fuller R, et al. Effect of an interleukin-4 variant on late phase asthmatic response to allergen challenge in asthmatic patients: results of two phase 2a studies. Lancet. 2007;370:1422-1431.

[37] van der Pouw Kraan TC, van der Zee JS, Moeije LC, et al. The role of IL-13 in IgE synthesis by allergic asthma patients. Clin Exp Immunol. 1998;111:129-135.

[38] Foster PS, Hogan SP, Ramsay AJ, et al. Interleukin 5 deficiency abolishes eosinophilia, airways hyperreactivity, and lung damage in a mouse asthma model. J Exp Med. 1996;183:195-201.

[39] Chung KF. Targeting the interleukin pathway in the treatment of asthma. Lancet. 2015;386:1086-1096.

[40] Singh A, Yamamoto M, Ruan J, et al. Th17/Treg ratio derived using DNA methylation analysis is associated with the late phase asthmatic response. Allergy Asthma Clin Immunol. 2014;10(32):1-9.

[41] Ying S, Robinson DS, Varney V, et al. TNF alpha mRNA expression in allergic inflammation. Clin Exp Allergy. 1991;21:745-750.

[42] Bradding P, Roberts JA, Britten KM, et al. Interleukin-4, -5 , and -6 and tumor necrosis factor-alpha in normal and asthmatic airways: evidence for the human mast cell as a source of these cytokines. Am J Respir Cell Mol Biol. 1994;10:471-480.

[43] Cho JY, Park S-C, Kim T-W, et al. Radical scavenging and anti-inflammatory activity of extracts from Opuntia humifusa Raf. J Pharm Pharmacol. 2006;58(1):113-119.

[44] Karym E-M, Youssef EK, Asmaa B, et al. Nopal Cactus (Opuntia humifusa-indica) as a source of bioactive compounds for nutrition, health and disease. Molecules. 2014;19:14879-14901.

[45] Jung CH, Lee JY, Cho CH, et al. Anti-asthmatic action of quercetin and rutin in conscious guinea-pigs challenged with aerosolized ovalbumin. Arch Pharm Res. 2007;30(12):1599-1607.

[46] Choi SB, Bae G-S, Park K-C, et al. Opuntia humifusa ameliorated cerulein-induced acute pancreatitis. Pancreas. 2014;43(1):118-127.

[47] Hahm S-W, Park J, Son Y-S. Opuntia humifusa stems lower blood glucose and cholesterol levels in streptozotocin-induced diabetic rats. Nutr Res. 2011;31(6):479-487. 